\title{
Transcriptome analysis reveals molecular profiles associated with evolving steps of monoclonal gammopathies
}

\author{
Lucía López-Corral, ${ }^{1}$ Luis Antonio Corchete, ${ }^{1}$ María Eugenia Sarasquete, ${ }^{1}$ María Victoria Mateos, ${ }^{1}$ Ramón García-Sanz, ${ }^{1}$ \\ Encarna Fermiñán, ${ }^{2}$ Juan-José Lahuerta, ${ }^{3}$ Joan Bladé, ${ }^{4}$ Albert Oriol, ${ }^{5}$ Ana Isabel Teruel, ${ }^{6}$ María Luz Martino, ${ }^{7}$ \\ José Hernández, ${ }^{8}$ Jesús María Hernández-Rivas, ${ }^{1}$ Francisco Javier Burguillo, ${ }^{9}$ Jesús F. San Miguel, ${ }^{1}$ \\ and Norma C. Gutiérrez ${ }^{1}$
}

\begin{abstract}
${ }^{1}$ Servicio de Hematología del Hospital Universitario de Salamanca, IBSAL IBMCC (USAL-CSIC), Salamanca; ${ }^{2}$ Centro de Investigación del Cáncer-IBMCC (USAL-CSIC), Salamanca; ${ }^{3}$ Servicio de Hematología del Hospital 12 de Octubre, Madrid; ${ }^{4}$ Servicio de Hematología del Hospital Clinic, Barcelona; ${ }^{5}$ Servicio de Hematología del Hospital Germans Trias i Pujol, Badalona; ${ }^{6}$ Servicio de Hematología del Hospital Universitario de Valencia; ${ }^{7}$ Servicio de Hematología del Hospital Virgen del Rocío, Sevilla; ${ }^{8}$ Servicio de Hematología de Hospital General de Segovia; and ${ }^{9}$ Departamento de Química Física, Facultad de Farmacia, Universidad de Salamanca, Spain
\end{abstract}

A multistep model has been proposed of disease progression starting in monoclonal gammopathy of undetermined significance continuing through multiple myeloma, sometimes with an intermediate entity called smoldering myeloma, and ending in extramedullary disease. To gain further insights into the role of the transcriptome deregulation in the transition from a normal plasma cell to a clonal plasma cell, and from an indolent clonal plasma cell to a malignant plasma cell, we performed gene expression profiling in 20 patients with monoclonal gammopathy of undetermined significance, 33 with high-risk smoldering myeloma and 41 with multiple myeloma. The analysis showed that 126 genes were differentially expressed in monoclonal gammopathy of undetermined significance, smoldering myeloma and multiple myeloma as compared to normal plasma cell. Interestingly, 17 and 9 out of the 126 significant differentially expressed genes were small nucleolar RNA molecules and zinc finger proteins. Several proapoptotic genes (AKT1 and AKT2) were down-regulated and antiapoptotic genes (APAF1 and BCL2L1) were upregulated in multiple myeloma, both symptomatic and asymptomatic, compared to monoclonal gammopathy of undetermined significance. When we looked for those genes progressively modulated through the evolving stages of monoclonal gammopathies, eight snoRNA showed a progressive increase while APAF1, VCAN and MEGF9 exhibited a progressive downregulation. In conclusion, our data show that although monoclonal gammopathy of undetermined significance, smoldering myeloma and multiple myeloma are not clearly distinguishable groups according to their gene expression profiling, several signaling pathways and genes were significantly deregulated at different steps of the transformation process.

\section{Introduction}

Multiple myeloma (MM) is a plasma cell malignancy characterized by high genetic complexity. ${ }^{1}$ The development of high-throughput methodologies for genomic analysis has greatly increased our understanding of the pathogenesis of MM. These techniques show that genetic heterogeneity ultimately results in different gene expression profiles specific to singular genetic abnormalities. Thus, the recurrent IGH translocations, the most frequent gains and losses, as well as the hyperdiploid and non-hyperdiploid categories display specific expression signatures. ${ }^{2-}$

Based on clinical and biological data, a multistep model of disease progression has been proposed, starting in monoclonal gammopathy of undetermined significance (MGUS) continuing through $\mathrm{MM}$, sometimes with an intermediate entity called smoldering $\mathrm{MM}(\mathrm{SMM})$, and ending in extramedullary disease. ${ }^{8-11}$ Cytogenetic studies using fluorescence in situ hybridization analysis (FISH) and more sophisticated genomic technologies, such as SNP-based mapping arrays and whole genome sequencing have provided a framework of the recurrent chromosomal abnormalities associated with the evolving stages of monoclonal gammopathies, and highlighted that most genetic lesions typical of $\mathrm{MM}$ are already present in MGUS and SMM steps. ${ }^{12-16}$ We have shown that a major difference among these three entities is the number of clonal PC with genetic abnormalities that increases from MGUS to SMM and to MM, suggesting a clonal expansion of genetically abnormal PC. ${ }^{17,18}$ Regarding progression genetic markers, it has been recently shown that specific chromosomal abnormalities, such as del(17p), t(4;14), 1q gains and hyperdiploidy significantly predict progression to active MM in SMM patients. ${ }^{19,20}$

GEP has also been used for identifying genes deregulated in the transition from normal plasma cells (NPC) to MGUS and $M M{ }^{6,10,21-23}$ In this setting, it has been shown that the number of genes differentially expressed between NPC and MGUS is significantly higher than those deregulated between MGUS and $M M{ }^{10,22}$ although these two diseases could be differentiated based on expression of genes mainly involved in metab- 
olism and proliferation. ${ }^{6}$ Regarding SMM, there has been no attempt to define an expression signature able to discriminate asymptomatic and symptomatic MM. Probably, the blurred limit between the established criteria for distinguishing MGUS, SMM and MM hampers the identification of characteristic expression patterns of the multistep transformation process.

Our aim was to gain further insights into the transcriptome deregulation observed in clonal PC from MGUS, SMM and symptomatic MM compared to NPC, and in addition, to find out whether the different evolving steps of monoclonal gammopathies display a singular expression signature.

\section{Methods}

\section{Patients and samples}

Bone marrow (BM) samples were obtained from 20 patients with MGUS, 33 with high-risk SMM and 41 with MM. All samples corresponded to newly diagnosed untreated patients. To avoid misclassification or overlapping entities, we decided to focus on MGUS patients with more than two years of stable follow up. Samples were classified according to the International Myeloma Working Group criteria. ${ }^{24}$ The criteria for defining high-risk SMM has been described previously. ${ }^{18,25}$ In addition, 5 healthy donors were also included in order to relate the deregulation of gene expression profiling of clonal populations to normal condition. The study was approved by the research ethics committees of all participating institutions and written informed consent was obtained from all patients in accordance with the Declaration of Helsinki. The main clinical and laboratory characteristics of these patients are shown in Online Supplementary Table S1. The FISH, SNP arrays and GEP of the patients with high-risk SMM as well as FISH analysis of the remaining samples have been previously reported. ${ }^{17,26}$ The frequency of the genetic abnormalities of the patients included in the present study is indicated in Online Supplementary Table S2.

In all the samples, a CD138-positive PC isolation using the AutoMACs separation system (Miltenyi-Biotec) was carried out. Final purity was over $95 \%$ in all MM and SMM cases, and over $90 \%$ in MGUS patients and healthy donors.

\section{RNA extraction and microarray hybridization}

Total RNA was extracted using the RNeasy Mini Kit according to the manufacturer's instructions. The RNA integrity was assessed using Agilent 2100 Bioanalyzer (Agilent). RNA labeling and microarray hybridization to Human Gene 1.0 ST Array (Affymetrix) have been previously reported. ${ }^{27}$ Full microarray data are available at the Gene Expression Omnibus (www.ncbinlm.nih.gov/geol, accession n: GSE47552).

\section{Gene expression data analysis Data pre-processing}

Data pre-processing: normalization was carried out by using the expression console (Affymetrix) with RMA algorithm which includes background correction, normalization and calculation of expression values $\left(\log _{2}\right) \cdot{ }^{28}$ Since myeloid contamination signature can be detected even in samples with high purity, those probes identifying genes exclusive of myeloid lineage were subtracted from the analysis from the outset (Online Supplementary Table S3).

\section{Unsupervised analysis}

The package pvclust (http://www.is.titech.ac.jp/shimo/prog/pvclust/) was used for the calculations of hierarchical clustering analysis with $P$ values for all clusters, using the Euclidean distance as distance measure and the group average link method. The hypothesis that "the cluster does not exist" was rejected with a significance level of 0.05 .

\section{Supervised analysis}

Significant Analysis of Microarrays (SAM) algorithm (http://www-stat.standford.edu/-tibs/SAM) was used to identify genes with statistically significant changes in expression between different classes. ${ }^{29}$ All data were permutated over 1000 cycles by using the two-class and multiclass response format, without considering equal variances. Significant genes were selected based on the lowest q-value (q-value $<10^{-5}$ ).

\section{Gene function analysis}

The probe sets were functionally annotated and grouped according to their biological function using Gene Ontology biological process descriptions. The functional analysis to identify the most relevant biological mechanisms, pathways and functional categories in the data sets of genes selected by statistical analysis, was generated through the use of IPA (Ingenuity Systems, www.ingenuity.com).

\section{Comparative analysis with other gene expression datasets}

In order to validate the supervised analysis of the present gene expression data, two datasets including NPC, MGUS, SMM and $\mathrm{MM}$ samples were generated from records of three series belonging to two different institutions publicly available at Gene Expression Omnibus (GEO, www.ncbi.nlm.nih.gov/geo/): GEO GSE5900 and GSE2658 (UAMS: NPC=22, MGUS=44, SMM=12 and $M M=256$ ) and GEO GSE6477 (Mayo Clinic: NPC=15, $M G U S=22, S M M=24$ and $M M=73$ ). The approaches used for comparative analysis are detailed in the Online Supplementary Appendix.

\section{Quantitative real-time PCR}

Methodology is described in the Online Supplementary Appendix.

\section{Results}

In a first approach to the gene expression data set, we explored the separation of the samples into clusters based on similarities in gene expression by using unsupervised hierarchical clustering. Despite the fact that the four sample classes were not clearly grouped in separated branches, the five NPC samples were clustered next to one another and included in a subbranch (Figure 1).

Then, we investigated whether the selected PC population from monoclonal gammopathy patients displayed specific expression profiles that were clearly distinguishable from PC from healthy donors. In order to avoid an imbalance in the analysis when comparing 5 NPC with more than 90 samples of clonal PC, and to avoid a potential selection of genes strongly deregulated in only one of the groups and therefore significant in the comparison with NPC, we considered it more appropriate to compare separately each of the three entities with the group of NPC to subsequently cross the three comparisons and select the intersection genes. Finally, we looked for genes deregulated in MGUS compared to MM, SMM compared to MM and MGUS compared to SMM, in order to identify gene categories and molecular pathways involved in MM development. Since SMM category included high-risk 
asymptomatic patients, we also investigated the common deregulated genes in SMM and MM patients compared to the indolent MGUS state.

\section{Expression signature of clonal PC related to normal status}

We identified a set of 126 common genes differentially expressed in MGUS, SMM and MM as compared to NPC $\left(q\right.$-value $\left.<10^{-5}\right)$, the majority of them (106 genes) downregulated in clonal PC (Online Supplementary Table S4). Further functional analysis of these 126 genes showed that the two most significant molecular and cellular functional categories were those related with cell cycle (ATF2, GADD45A, CDC6 and IL-16) and cell death (BCL2L1, CD81, CDC6, IGHM, ABCC1, ATF2 and ATXN2) $(P<0.01)$.

On the other hand, the top three canonical pathways (well characterized metabolic and cell signaling pathways) were EIF2 signaling, regulation of EIF4 and p70S6K signaling, and mTOR signaling $(P<0.001)$. These pathways play critical roles in translational regulation and the most differentially expressed genes contained in them belonged to ribosomal protein family. Other relevant canonical pathways involved in the transition from NPC to clonal PC were several interleukin signaling pathways, B-cell development, glucocorticoid receptor signaling, G2/M DNA damage checkpoint regulation and ATM, ERK5, VEGF, P53 and SAPK/JNK signaling pathways $(P<0.05)$.

Interestingly, 17 and 9 out of the 126 significant differentially expressed genes were small nucleolar RNA molecules (snoRNA) and zinc finger proteins, respectively. Both groups of genes were down-regulated in the comparison between healthy PC and monoclonal gammopathies PC (Figure 2).

When we looked at the top 10 up-regulated and downregulated genes, we found important genes involved in cancer pathogenesis (Figure 3). The most significant under-expressed gene in clonal PC compared to NPC was $C A B L E S$ and the most significant up-regulated gene was
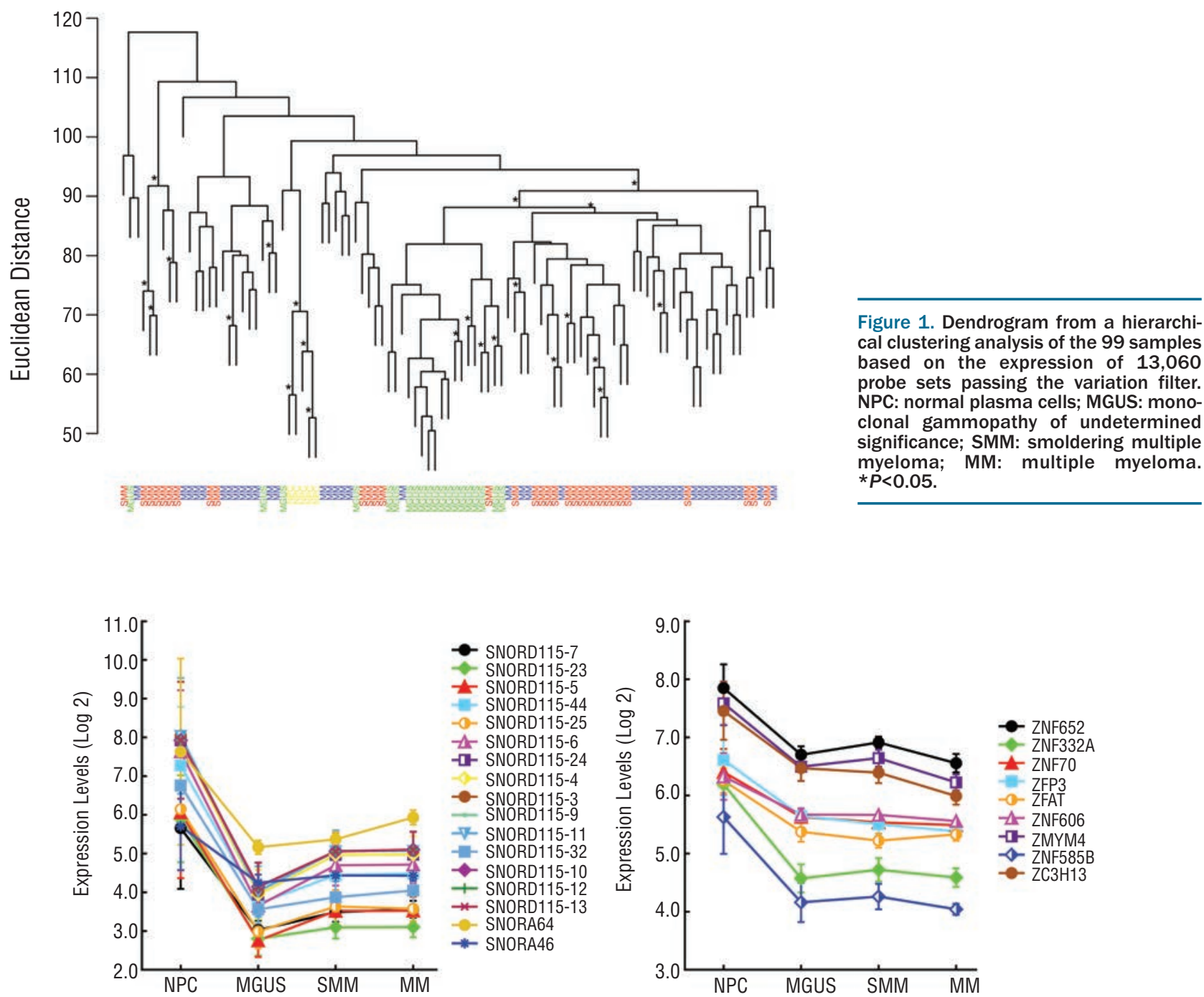

Figure 2. Expression levels of several small nucleolar RNA and zinc finger proteins in plasma cells from healthy donors, MGUS, SMM and MM patients. 
GADD45A. It is important to note that 5 (RPS28, RPS10, RPS17, RPS12 and RPS27A) of the top 10 up-regulated genes belonged to ribosomal protein family. TMSB10 gene, also up-regulated in clonal PC, is associated with tumor progression and metastasis in several cancers through JNK activation. ${ }^{30}$

Genes differentially expressed between MGUS and MM

A total of 1184 genes were differentially expressed between MGUS and MM samples (642 genes were downregulated and 542 were up-regulated in $\mathrm{MM}$ ). The functional analysis revealed that the molecular and cellular function the most significantly affected in the comparison between MGUS and MM was cell death, with 106 genes involved $(P<0.01)$. When the genes included in this category were evaluated according to their anti or proapoptotic role, an imbalance in favor of antiapoptotic state was observed in MM. Thus, AKT1 and AKT2, antiapoptotic genes, were over-expressed in MM compared to MGUS. In fact, (PI3K)/Akt pathway has been reported to contribute to the malignant growth of $\mathrm{MM} .{ }^{31}$ The upregulation of $B A G 3$ and GADD $45 B$ in MM also contribute to the antiapoptotic state of this disease. ${ }^{32,33}$ Moreover, APAF1
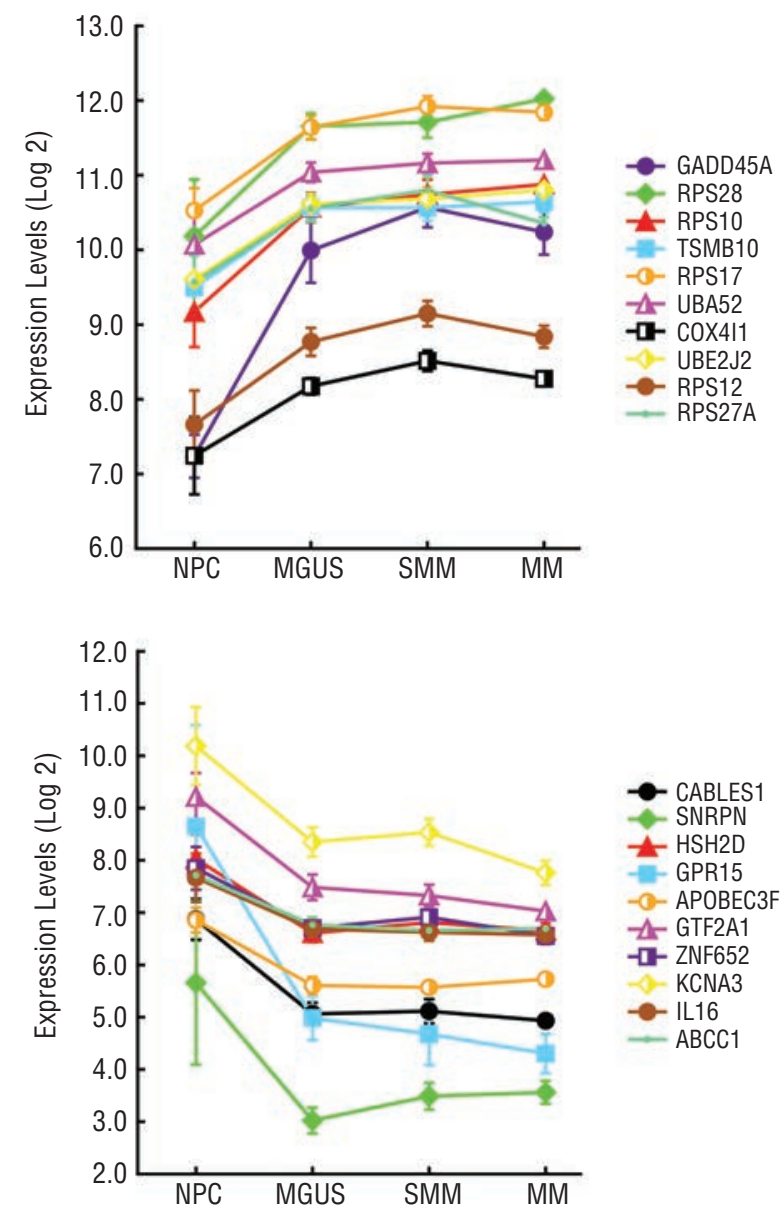

Figure 3. Expression of the top 10 up-regulated and down-regulated genes from the comparison between clonal plasma cells and normal plasma cells. (one of the most down-regulated genes with an FC of 6.9) and BCL2L1, which are apoptotic activators, were downregulated in $\mathrm{MM}$ as compared to MGUS. ${ }^{34,35}$

The canonical pathway most statistically significant and with the highest number of genes corresponded to EIF2, which catalyzes the first regulated step of protein synthesis initiation. ${ }^{36}$ Several ribosomal proteins were included in this pathway. IL-8 signaling was also at the top of the most significant canonical pathway list and, interestingly, another five significant canonical pathways involved interleukins (IL-8, IL-17A, IL-6, IL-15, IL-10 and IL-4). One of the top 10 genes up-regulated in MM compared to MGUS was TERC.

\section{Genes differentially expressed between SMM and MM}

In the SMM versus MM comparison, 1163 genes were significantly deregulated (936 genes were down-regulated and 227 were up-regulated in MM). DNA replication, recombination and repair, containing 16 genes, were the most significant deregulated molecular and cellular function. ATM and RAD17, important cell cycle checkpoint genes, together with $R A D 50$, which are directly involved in DNA damage repair, were included in this molecular function. ${ }^{37}$
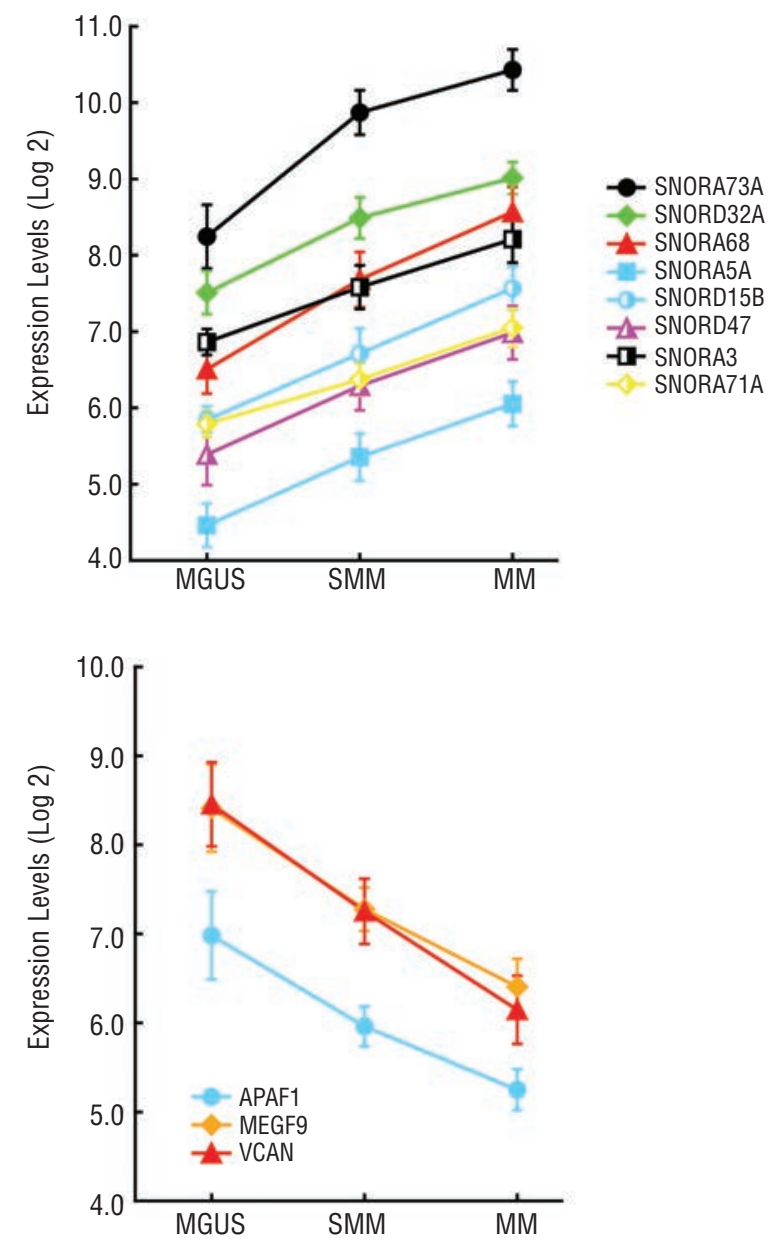

Figure 4. Expression of the genes significantly deregulated among the three entities. 
Telomere extension by telomerase, Myc mediated apoptosis, insulin receptor, IGF1, p53, NF-kB, TGFb and Jak/stat signaling were included within the most significant canonical pathways. Myc mediated apoptosis signaling is of particular interest, as it is frequently associated with human malignancies, including $M M^{21}$ This pathway involved genes like the proapoptotic $B A D$, the adapter molecule for the death receptor family FADD and caspase 8, among others. ${ }^{35}$

\section{Genes differentially expressed between MGUS and SMM}

The analysis showed that a set of 627 differentially expressed genes was able to differentiate MGUS from SMM $\left(q\right.$-value $\left.<10^{-5}\right)$ (237 genes were down-regulated in SMM and 390 were up-regulated). Similarly to the comparison between MGUS and MM, cell death was identified as the most significant molecular function affected in the transition from MGUS to SMM, based on the deregulation of 55 genes $(P<0.01)$. For example, the proapoptotic gene APAF1 was down-regulated in SMM patients and the antiapoptotic genes BAG1 and BAG3 were over-expressed in SMM.

The RAC and NFKB signaling $(P<0.01)$ were included within the top canonical pathways. RELA, a NFKB component, is a pleiotropic transcription factor involved in many biological processes, such as cell growth, tumorigenesis and apoptosis. ${ }^{38}$ Within the top 10 down-regulated genes, $E R G$ was one of the most under-expressed genes in SMM. ERG is a key regulator of cell proliferation and differentiation, and angiogenesis. ${ }^{39}$ Regarding the top 10 overexpressed genes, RPL7A is a ribosomal protein and an altered pattern of RPL7 expression has been described in colorectal carcinoma. ${ }^{40}$

\section{Genes differentially expressed between MGUS and myeloma disease (SMM/MM)}

After analyzing the gene expression differences between MGUS, SMM and MM, and since high-risk smoldering myeloma is an entity very close to symptomatic myeloma, we wanted to focus on those genes that characterize the myeloma disease compared to the indolent MGUS state. A set of 275 genes commonly deregulated in SMM and MM was able to distinguish the myeloma disease (SMM and MM) from MGUS $\left(q\right.$-value $\left.<10^{-5}\right)$.

Cell death and survival was identified as the most significant molecular function affected in the transition from MGUS to myeloma disease, based on the deregulation of 35 genes $(P<0.01)$, such as the antiapoptotic gene $B A G 3$ and the NFKB component RELA (both up-regulated in SMM and MM). Similar to a comparison of MGUS versus SMM, the RAC signaling pathway deregulation $(P<0.01)$ was included within the five most significant canonical pathways (RELA, NFKBIA, MAPK1, CEBPD, LCN2 and IL17RA).

\section{Genes with progressive modulation from MGUS to SMM and to MM}

Finally, we also looked for those genes significantly deregulated among the three entities, which in turn were progressively up- or down-regulated from MGUS to SMM and to $\mathrm{MM}$. We reasoned that these genes could be the most significant for promoting multistep transformation.

Surprisingly, only 11 out of the 2335 significant genes exhibited a progressive deregulation $(P<0.0001)$ in the evolving stages of monoclonal gammopathies. All the genes with a progressive increase from MGUS to SMM and to MM were small nucleolar RNA genes (snoRNA) (Figure 4). In addition, only 3 genes, APAF1, VCAN and MEGF9, showed a progressive downregulation in the transition from MGUS to SMM and to MM (Figure 4). All the 3 genes have been described to be involved in cancer: APAF1 encodes a cytoplasmic protein that initiates apoptosis, and decreased expression of this gene has been reported in malignant PC compared to $\mathrm{NPC} ;{ }^{41}$ VCAN deregulation has been shown during the malignant transformation and tumor progression; ${ }^{42}$ and MEGF9, an epidermal growth factor involved in cell adhesion, receptorligand interactions and tissue repairs, has been related to local aggressiveness and soft tissue tumor. ${ }^{43}$

\section{Comparison of expression signatures to independent monoclonal gammopathy datasets}

In order to compare the gene expression signatures generated in the present study with previously published data, we carried out three analyses. First, we performed a semi-supervised approach whereby a set of genes significantly deregulated in a multiclass comparison, including NPC, MGUS, SMM and MM samples from the present study, was subsequently used for identifying these subgroups in UAMS and Mayo Clinic datasets. As shown in Online Supplementary Figure S1, NPC, MGUS and MM samples showed a trend to group in separated clusters, while SMM patients were intermingled with the other samples.

Then, we compared the genes differentially expressed in MGUS, SMM and MM with regard to NPC in the three datasets. Using a less stringent supervised analysis ( $q$-value $<0.05$ ), a set of 4067, 2089 and 1583 genes differentially expressed in clonal PC related to normal status were found in the UAMS, Mayo Clinic and the present studies, respectively. A total of 742 out of the 1583 (47\%) deregulated genes in our study were found to be significantly altered in the UAMS and/or Mayo Clinic dataset analysis (Online Supplementary Figure 2A). Further functional analysis of the differentially expressed genes showed that cell death and survival were the most significant molecular and cellular functional categories in the three datasets based on the deregulation of more than 300 genes. Interestingly, EIF2 signaling pathway was the most significant canonical pathway $(P<0.001)$ deregulated between clonal and NPC, both in Mayo Clinic samples and in the present study.

Finally, we sought to identify genes and molecular pathways commonly deregulated in the three studies when comparing MGUS with MM. A total of 2627 out of the $3570(74 \%)$ deregulated genes in our study were also aberrantly expressed in Mayo Clinic and/or UAMS dataset analysis (Online Supplementary Figure 2B). The functional analysis showed that cell death and survival, and cellular growth and proliferation were the two most significant molecular and cellular functional categories in the three datasets, based on the deregulation of more than 700 genes $(P<0.05)$. Among the five top canonical pathways, EIF2 signaling and mitochondrial dysfunction were significantly altered in the Mayo Clinic and our studies.

\section{Validation of gene expression using $q R T-P C R$}

In order to validate some of the results, 10 genes (APAF1, VCAN, MEGF9, SNORD55, TPI1, TERC, BAG1, BAD, RPLTA and AKT1), selected according to their intensity 
values and biological relevance, were analyzed by qRTPCR in 14 MGUS, 10 SMM and 10 MM samples (Online Supplementary Figure S3). Seven out of the 10 genes showed statistically significant differential expression between the pre-malignant conditions and symptomatic $\mathrm{MM}$, with up- or downregulation in agreement with microarray results. APAF1, VCAN and MEGF9 showed significant downregulation in the transition from MGUS to SMM and to MM, while BAD and TERC were significantly up-regulated in the comparison between MGUS and MM. In addition, SNORD 55 and TPI1 showed progressive overexpression from MGUS to SMM and to MM samples.

\section{Discussion}

Gene expression profiling is a robust approach for the accurate identification of molecular subtypes of MM. However, the molecular definition of the different evolving steps of monoclonal gammopathies is not so clear. ${ }^{6,23}$ This is the first report that includes a large number of patients with SMM in order to tackle the GEP analysis of the different clonal plasma cells involved in the multistep evolution process from NPC to symptomatic MM.

Consistent with previous reports, the comparison between normal and clonal PC from MGUS, SMM and $\mathrm{MM}$ demonstrates a clear predominance of down-regulated genes. ${ }^{10}$ Notably, the largest group of genes down-regulated in clonal PC represented small nucleolar RNAs (snoRNAs). snoRNA are non-coding RNAs (ncRNA) that are less well known than other ncRNAs, such as miRNAs and siRNAs, that could be actively involved in the development of cancer. ${ }^{44.47}$ In this setting, global downregulation is a characteristic feature of snoRNAs in cancer cells. For example, it has been recently reported that leukemic cells show infraexpression of several snoRNAs. ${ }^{47}$ In addition, 9 out of 126 significant differentially expressed genes in clonal PC were zinc finger proteins, which are a family of transcription factors involved in governing the response to growth factor signaling. ${ }^{10,48-50}$ CABLES1, which is a cyclindependent kinase (CDK)-binding protein that plays an inhibitor role in proliferation and/or differentiation and whose loss of expression has been found in many human cancers, ${ }^{51,52}$ was the most under-expressed gene in clonal PC versus NPC. By contrast, GADD 45B, which encodes a protein, and which responds to environmental stresses by mediating activation of the p38/JNK pathway via MTK1/MEKK4 kinase, was the most significant up-regulated gene in clonal PC. The DNA damage-induced transcription of this gene is mediated by both p53-dependent and $\mathrm{p} 53$-independent mechanisms. ${ }^{32}$

On the other hand, our results found a large number of genes differentially expressed between MGUS and MM patients, which is not consistent with a previous report showing that most gene expression changes occur between NPC and MGUS with less than 100 genes differentially expressed between MGUS and MM. ${ }^{10}$ Probably, the number of cases analyzed and the new version of Affymetrix array used in our study, which interrogates approximately 28,000 well-annotated genes, could explain these differences.

Since MM is a hypoproliferative tumor, it is thought that defects in apoptosis are potentially more important in MM pathogenesis than deregulation of genes involved in cell growth and proliferation. This is in agreement with the fact that several proapoptotic genes were down-regulated and antiapoptotic genes up-regulated in MM, both symptomatic and asymptomatic, compared to MGUS. Thus, AKT1 and AKT2 were found to be over-expressed in MM versus MGUS, which may contribute to the inactivation of the apoptosis signaling observed in the myeloma group. This finding is consistent with the contribution of PI3K/Akt pathway to the malignant growth of MM previously described. ${ }^{31}$ Moreover, BAG1 and BAG3 were also over-expressed in SMM versus MGUS. Members of the BAG family are cytoprotective proteins that bind to and regulate HSP70 family molecular chaperones. BAG1 is a multifunctional protein involved in different cell processes linked to cell survival. It has been reported as deregulated in diverse cancer types, including hematology tumors. ${ }^{53}$ BAG3 is constitutively expressed in a variety of tumors, including pancreas carcinomas, lymphocytic and myeloblastic leukemias, and thyroid carcinomas. ${ }^{33}$ Moreover, it has been reported that downregulation of BAG3 in vivo, which results in cell death, could be a potential anticancer therapeutic strategy. ${ }^{33}$ In addition, one of the top 10 genes up-regulated in MM versus MGUS was TERC. Deregulation of telomerase expression in somatic cells may be involved in oncogenesis. ${ }^{54}$ Regarding plasma cell dyscrasias, it has been reported that MGUS patients exhibit normal levels of telomerase activity in their PC, while the majority of $\mathrm{MM}$ and plasma cell leukemias express high levels of telomerase activity. This finding supports the hypothesis that activation of telomerase could play a role in the malignant transformation from MGUS to MM. ${ }^{55}$

Recently, an activation of MYC signature has been suggested to be involved in the transformation from MGUS to $M M$, since this signature was expressed in approximately $67 \%$ of MM while it was not present in NPC or MGUS. ${ }^{21}$ Consistent with this finding, we have shown that Myc-mediated apoptosis signaling is one of the top canonical pathways differentiating the asymptomatic and symptomatic myeloma.

We have also analyzed the genes which showed a progressive modulation from MGUS to SMM and to MM because we hypothesized that they could be directly involved in the multistep transformation of monoclonal gammopathies. However, a very small group of genes with a significant progressive deregulation in the evolving stages of the monoclonal gammopathies was found. Interestingly, all the genes with a progressive increase were snoRNA. Within the genes showing a progressive downregulation, APAF1 has been previously reported to be decreased in myeloma cells compared to NPC. ${ }^{41}$ The apoptotic peptidase activating factor 1 (APAF1) is a gene that encodes a cytoplasmic protein that initiates apoptosis through activating caspase 9 . Activated caspase 9 stimulates the subsequent caspase cascade that commits the cell to apoptosis. ${ }^{41}$ Therefore, further investigation of the potential role of this gene on the transformation process is warranted.

The GEP in MM are significantly related to genetic abnormalities. Therefore, a different distribution of IGH tx, 13q deletion and 1q gains in the MGUS, SMM and MM groups could affect their transcriptional profile. In the present study, the distribution according to genetic abnormalities was apparently similar for the three entities, although the small number of patients has to be taken into account. 
Finally, two approaches were carried out in order to validate our data: a comparative analysis using our dataset and two publicly available datasets obtained from two independent groups, and a validation of the expression levels of 10 of the most relevant genes by qRT-PCR. Regarding validation with external data, the semi-supervised analysis performed particularly well, grouping most of NPC together and distinguishing clusters that contained mostly MM or mainly MGUS. However, the different criteria used for selecting SMM samples in the three series prevented the clustering of these cases. When the differentially expressed genes were compared, the number of commonly deregulated genes was higher in the comparison between MGUS and MM than between NPC and clonal PC from all gammopathy stages. The heterogeneity of the latter group probably contributes to such a difference. Importantly, the EIF2 signaling pathway was recurrently selected in the three datasets as one of the most significantly deregulated canonical pathways. This finding substantiates previous observations pointing out the potential relevance of this network in MM pathogenesis, such as a novel therapeutic approach to maximize bortezomib-induced apoptosis based on the inhibition of eIF2A dephosphorylation. ${ }^{56}$ Additionally, the technical validation by qRT-PCR of APAF1, VCAN, MEGF9, SNORD55, TPI1, $T E R C, B A D$ expression showed consistent results with the expression levels assessed by microarrays. This confirmation supports further investigation of the functional role of these genes in the gammopathy transformation process.

In conclusion, our data show that although MGUS, SMM and MM are not clearly distinguishable groups according to their GEP, there were several signaling pathways and genes deregulated in the different steps of transformation process. The functional analysis of some of these deregulated genes with more biological significance would be of great importance to understand the bases underlying the transformation from MGUS and SMM to symptomatic MM.

\section{Acknowledgments}

The authors would like to thank "Grupo Español de Mieloma" clinicians for providing $M M$ samples, and S. González, I. Rodriguez, I. Isidro, T. Prieto and V. Gutiérrez for technical assistance.

\section{Funding}

This study was partially supported by Spanish FIS (PI080568, PS09/01450 and PS0901897), "Gerencia Regional de Salud, Junta de Castilla y León" (GRS 702/A/11) grant, and the Spanish Myeloma Network Program (RD06/0020/0006, $R D 12 / 0036 / 0058$ and RD12/0036/0046).

\section{Authorship and Disclosures}

Information on authorship, contributions, and financial \& other disclosures was provided by the authors and is available with the online version of this article at www. haematologica.org.

\section{References}

1. Morgan GJ, Walker BA, Davies FE. The genetic architecture of multiple myeloma. Nat Rev Cancer. 2012;12(5):335-48

2. Agnelli L, Bicciato S, Mattioli M, Fabris S, Intini $\mathrm{D}$, Verdelli $\mathrm{D}$, et al. Molecular classification of multiple myeloma: a distinct transcriptional profile characterizes patients expressing CCND1 and negative for 14q32 translocations. J Clin Oncol. 2005;23(29): 7296-306.

3. Bergsagel PL, Kuehl WM. Molecular pathogenesis and a consequent classification of multiple myeloma. J Clin Oncol. 2005;23 (26):6333-8.

4. Bergsagel PL, Kuehl WM, Zhan F, Sawyer J, Barlogie B, Shaughnessy J Jr. Cyclin D dysregulation: an early and unifying pathogenic event in multiple myeloma. Blood. 2005;106(1):296-303.

5. Chng WJ, Kumar S, Vanwier S, Ahmann G, Price-Troska T, Henderson K, et al. Molecular dissection of hyperdiploid multiple myeloma by gene expression profiling. Cancer Res. 2007;67(7):2982-9.

6. Mattioli M, Agnelli L, Fabris S, Baldini L, Morabito F, Bicciato S, et al. Gene expression profiling of plasma cell dyscrasias reveals molecular patterns associated with distinct IGH translocations in multiple myeloma. Oncogene. 2005;24(15):2461-73.

7. Xiong W, Wu X, Starnes S, Johnson SK, Haessler J, Wang S, et al. An analysis of the clinical and biologic significance of TP53 loss and the identification of potential novel transcriptional targets of TP53 in multiple myeloma. Blood. 2008;112(10):4235-46.

8. Landgren $\mathrm{O}$, Kyle RA, Pfeiffer RM,
Katzmann JA, Caporaso NE, Hayes RB, et al. Monoclonal gammopathy of undetermined significance (MGUS) consistently precedes multiple myeloma: a prospective study. Blood. 2009;113(22):5412-7.

9. Weiss BM, Abadie J, Verma P, Howard RS, Kuehl WM. A monoclonal gammopathy precedes multiple myeloma in most patients. Blood. 2009;113(22):5418-22.

10. Davies FE, Dring AM, Li C, Rawstron AC, Shammas MA, O'Connor SM, et al. Insights into the multistep transformation of MGUS to myeloma using microarray expression analysis. Blood. 2003; 102(13):4504-11.

11. Hallek M, Leif Bergsagel P, Anderson KC. Multiple Myeloma: Increasing Evidence for a Multistep Transformation Process. Blood. 1998;91(1):3-21.

12. Avet-Loiseau H, Li JY, Morineau N, Facon T, Brigaudeau C, Harousseau JL, et al. Monosomy 13 Is Associated With the Transition of Monoclonal Gammopathy of Undetermined Significance to Multiple Myeloma. Blood. 1999;94(8):2583-9.

13. Avet-Loiseau H, Facon T, Grosbois B, Magrangeas F, Rapp MJ, Harousseau JL, et al. Oncogenesis of multiple myeloma: 14q32 and $13 \mathrm{q}$ chromosomal abnormalities are not randomly distributed, but correlate with natural history, immunological features, and clinical presentation. Blood. 2002;99(6):2185-91.

14. Chiecchio L, Dagrada GP, Ibrahim AH, Dachs Cabanas E, Protheroe RKM, Stockley $\mathrm{DM}$, et al. Timing of acquisition of deletion 13 in plasma cell dyscrasias is dependent on genetic context. Haematologica. 2009;94 (12):1708-13

15. Fonseca R, Bailey RJ, Ahmann GJ, Rajkumar SV, Hoyer JD, Lust A, et al. Genomic abnor- malities in monoclonal gammopathy of undetermined significance. Blood. 2002;100 (4):1417-24.

16. Kaufmann H, Ackermann J, Baldia C Nosslinger T, Wieser R, Seidl S, et al. Both IGH translocations and chromosome $13 q$ deletions are early events in monoclonal gammopathy of undetermined significance and do not evolve during transition to multiple myeloma. Leukemia. 2004;18(11): 1879-82.

17. Lopez-Corral L, Gutierrez NC, Vidriales MB, Mateos MV, Rasillo A, Garcia-Sanz R, et al. The Progression from MGUS to Smoldering Myeloma and Eventually to Multiple Myeloma Involves a Clonal Expansion of Genetically Abnormal Plasma Cells. Clin Cancer Res. 2011;17(7):1692-700.

18. Lopez-Corral L, Sarasquete ME, Bea S, Garcia-Sanz R, Mateos MV, Corchete LA, et al. SNP-based mapping arrays reveal high genomic complexity in monoclonal gammopathies, from MGUS to myeloma status. Leukemia. 2012;26(12):2521-9.

19. Neben K, Jauch A, Hielscher T, Hillengass J, Lehners N, Seckinger A, et al. Progression in Smoldering Myeloma Is Independently Determined by the Chromosomal Abnormalities del(17p), $\mathrm{t}(4 ; 14)$, Gain 1q, Hyperdiploidy, and Tumor Load. J Clin Oncol. 2013;31(34):4325-32

20. Rajkumar SV, Gupta V, Fonseca R, Dispenzieri A, Gonsalves WI, Larson D, et al. Impact of primary molecular cytogenetic abnormalities and risk of progression in smoldering multiple myeloma. Leukemia. 2013;27(8):1738-44

21. Chng WJ, Huang GF, Chung TH, Ng SB, Gonzalez-Paz N, Troska-Price $\mathrm{T}$, et al Clinical and biological implications of MYC 
activation: a common difference between MGUS and newly diagnosed multiple myeloma. Leukemia. 2011;25(6):1026-35.

22. Zhan F, Hardin J, Kordsmeier B, Bumm K, Zheng $\mathrm{M}$, Tian $\mathrm{E}$, et al. Global gene expression profiling of multiple myeloma, monoclonal gammopathy of undetermined significance, and normal bone marrow plasma cells. Blood 2002;99(5):1745-57.

23. Zhan F, Barlogie B, Arzoumanian V, Huang Y, Williams DR, Hollmig K, et al. Geneexpression signature of benign monoclonal gammopathy evident in multiple myeloma is linked to good prognosis. Blood. 2007;109(4):1692-700.

24. International Myeloma Working Group. Criteria for the classification of monoclonal gammopathies, multiple myeloma and related disorders: a report of the International Myeloma Working Group. Br J Haematol. 2003;121(5):749-57.

25. Mateos MV, Hernandez MT, Giraldo P, de la Rubia J, de Arriba F, Lopez CL, et al. Lenalidomide plus dexamethasone for highrisk smoldering multiple myeloma. $\mathrm{N}$ Engl J Med. 2013;369(5):438-47.

26. Lopez-Corral L, Mateos MV, Corchete LA, Sarasquete MaE, de la Rubia J, de Arriba F, et al. Genomic analysis of high-risk smoldering multiple myeloma. Haematologica. 2012:97(9):1439-43.

27. Gutierrez NC, Sarasquete ME, MisiewiczKrzeminska I, Delgado M, De Las RJ, Ticona FV, et al. Deregulation of microRNA expression in the different genetic subtypes of multiple myeloma and correlation with gene expression profiling. Leukemia. 2010; 24(3):629-37.

28. Gutierrez NC, Ocio EM, De Las RJ, Maiso P, Delgado M, Ferminan E, et al. Gene expression profiling of $\mathrm{B}$ lymphocytes and plasma cells from Waldenstrom's macroglobulinemia: comparison with expression patterns of the same cell counterparts from chronic lymphocytic leukemia, multiple myeloma and normal individuals. Leukemia. 2007;21 (3):541-9.

29. Tusher VG, Tibshirani R, Chu G. Significance analysis of microarrays applied to the ionizing radiation response. Proc Natl Acad Sci USA. 2001;98(9):5116-21

30. Li M, Zhang Y, Zhai O, Feurino LW, Fisher WE, Chen C, et al. Thymosin beta-10 is aberrantly expressed in pancreatic cancer and induces JNK activation. Cancer Invest. 2009;27(3):251-6

31. Pene F, Claessens YE, Muller O, Viguie F, Mayeux P, Dreyfus F, et al. Role of the phosphatidylinositol 3-kinase/Akt and mTOR/P70S6-kinase pathways in the proliferation and apoptosis in multiple myeloma.
Oncogene. 2002;21(43):6587-97.

32. Liebermann DA, Hoffman B. Gadd45 in the response of hematopoietic cells to genotoxic stress. Blood Cells Mol Dis. 2007;39(3):329-35.

33. Cesaro E, Montano G, Rosati A, Crescitelli $\mathrm{R}$, Izzo P, Turco MC, et al. WT1 protein is a transcriptional activator of the antiapoptotic bag3 gene. Leukemia. 2010;24(6):1204-6.

34. Jourdan M, Reme T, Goldschmidt H, Fiol G, Pantesco V, De Vos J, et al. Gene expression of anti- and pro-apoptotic proteins in malignant and normal plasma cells. Br J Haematol. 2009;145(1):45-58.

35. Tarte K, Jourdan M, Veyrune JL, Berberich I, Fiol G, Redal N, et al. The Bcl-2 family member Bfl-1/A1 is strongly repressed in normal and malignant plasma cells but is a potent anti-apoptotic factor for myeloma cells. Br J Haematol. 2004;125(3):373-82.

36. Schmitt E, Naveau M, Mechulam Y. Eukaryotic and archaeal translation initiation factor 2: a heterotrimeric tRNA carrier FEBS Lett. 2010;584(2):405-12.

37. Shin MH, Yuan M, Zhang H, Margolick JB Kai M. ATM-dependent phosphorylation of the checkpoint clamp regulates repair pathways and maintains genomic stability. Cell Cycle. 2012;11(9):1796-803

38. Wertheimer E, Gutierrez-Uzquiza A Rosemblit C, Lopez-Haber C, Sosa MS, Kazanietz MG. Rac signaling in breast cancer: a tale of GEFs and GAPs. Cell Signal. 2012;24(2):353-62.

39. Sperone A, Dryden NH, Birdsey GM, Madden L, Johns M, Evans PC, et al. The transcription factor Erg inhibits vascular inflammation by repressing NF-kappaB activation and proinflammatory gene expression in endothelial cells. Arterioscler Thromb Vasc Biol. 2011;31(1):142-50.

40. Wang Y, Cheong D, Chan S, Hooi SC. Ribosomal protein L7a gene is up-regulated but not fused to the tyrosine kinase receptor as chimeric trk oncogene in human colorectal carcinoma. Int J Oncol. 2000;16(4):757-62.

41. Mitsiades N, Mitsiades CS, Poulaki V, Anderson KC, Treon SP. Intracellular regulation of tumor necrosis factor-related apoptosis-inducing ligand-induced apoptosis in human multiple myeloma cells. Blood. 2002;99(6):2162-71

42. Theocharis AD, Skandalis SS, Tzanakakis GN, Karamanos NK. Proteoglycans in health and disease: novel roles for proteoglycans in malignancy and their pharmacological targeting. FEBS J. 2010;277(19):3904-23.

43. Cunha IW, Carvalho KC, Martins WK Marques SM, Muto NH, Falzoni R, et al Identification of genes associated with local aggressiveness and metastatic behavior in soft tissue tumors. Transl Oncol. 2010;3(1): 23-32.

44. Mei YP, Liao JP, Shen JP, Yu L, Liu BL, Liu L, et al. Small nucleolar RNA 42 acts as an oncogene in lung tumorigenesis. Oncogene. 2011;31(22):2794-804

45. Dong XY, Guo P, Boyd J, Sun X, Li Q, Zhou $\mathrm{W}$, et al. Implication of snoRNA U50 in human breast cancer. J Genet Genomics. 2009:36(8):447-54.

46. Dong XY, Rodriguez C, Guo P, Sun X, Talbot JT, Zhou W, et al. SnoRNA U50 is a candidate tumor-suppressor gene at 6q14.3 with a mutation associated with clinically significant prostate cancer. Hum Mol Genet. 2008;17(7):1031-42

47. Valleron W, Laprevotte E, Gautier EF, Quelen C, Demur C, Delabesse E, et al. Specific small nucleolar RNA expression profiles in acute leukemia. Leukemia. 2012;26(9):2052-60

48. Yang L, Wang H, Kornblau SM, Graber DA Zhang N, Matthews JA, et al. Evidence of a role for the novel zinc-finger transcription factor ZKSCAN3 in modulating Cyclin D2 expression in multiple myeloma. Oncogene. 2011;30(11):1329-40

49. La Rocca R, Fulciniti M, Lakshmikanth $T$ Mesuraca M, Ali TH, Mazzei V, et al. Early hematopoietic zinc finger protein prevents tumor cell recognition by natural killer cells. J Immunol. 2009;182(8):4529-37.

50. Gyory I, Fejer G, Ghosh N, Seto E, Wright KL. Identification of a functionally impaired positive regulatory domain I binding factor 1 transcription repressor in myeloma cell lines. J Immunol. 2003; 170(6):3125-33.

51. Tan D, Kirley S, Li O, Ramnath N, Slocum $\mathrm{HK}$, Brooks JS, et al. Loss of cables protein expression in human non-small cell lung cancer: a tissue microarray study. Hum Pathol. 2003;34(2):143-9.

52. Zhang H, Duan HO, Kirley SD, Zukerberg LR, Wu CL. Aberrant splicing of cables gene, a CDK regulator, in human cancers. Cancer Biol Ther. 2005;4(11):1211-5.

53. Tsukahara F, Maru Y. Bag1 directly routes immature BCR-ABL for proteasomal degradation. Blood. 2010;116(18):3582-92.

54. Hahn WC. Role of telomeres and telomerase in the pathogenesis of human cancer. J Clin Oncol. 2003;21(10):2034-43.

55. Xu D, Zheng C, Bergenbrant S, Holm G Bjorkholm M, Yi Q, et al. Telomerase activity in plasma cell dyscrasias. Br J Cancer 2001;84(5):621-5.

56. Schewe DM, Aguirre-Ghiso JA. Inhibition of eIF2alpha dephosphorylation maximizes bortezomib efficiency and eliminates quiescent multiple myeloma cells surviving proteasome inhibitor therapy. Cancer Res. 2009;69(4):1545-52. 